OPEN ACCESS

Edited by:

Yusuke Moriguchi,

Kyoto University, Japan

Reviewed by:

Jeffrey Coldren,

Youngstown State University,

United States

Xiao Pan Ding

National University of Singapore,

Singapore

${ }^{*}$ Correspondence:

Liqi Zhu

zhulq@psych.ac.cn

Specialty section: This article was submitted to

Developmental Psychology, a section of the journal

Frontiers in Psychology

Received: 11 June 2017 Accepted: 02 October 2017 Published: 17 October 2017

Citation:

Yang X, Chen Z, Wang Z and Zhu L

(2017) The Relations between Television Exposure and Executive Function in Chinese Preschoolers: The Moderated Role of Parental Mediation Behaviors.

Front. Psychol. 8:1833. doi: 10.3389/fpsyg.2017.01833

\section{The Relations between Television Exposure and Executive Function in Chinese Preschoolers: The Moderated Role of Parental Mediation Behaviors}

\author{
Xiaohui Yang ${ }^{1,2}$, Zhe Chen ${ }^{3}$, Zhenhong Wang ${ }^{2}$ and Liqi Zhu ${ }^{1 *}$ \\ ${ }^{1}$ CAS Key Laboratory of Behavioral Science, Institute of Psychology, Chinese Academy of Sciences, Beijing, China, ${ }^{2}$ School \\ of Psychology, Shaanxi Normal University, Xi'an, China, ${ }^{3}$ Department of Human Ecology, University of California, Davis, \\ Davis, CA, United States
}

The present study examined the relations between preschoolers' television exposure and executive functions (EF). One hundred and nineteen 3- to 6-year-old children and their parents participated. Parents filled in a questionnaire regarding children's television viewing time, television content and parental mediation behaviors about their child's television viewing. The children were asked to finish six EF tasks, including the backward digit span task, the spatial span task, the boy-girl Stroop, the Simon task, the flanker task and the Tower of Hanoi task that assessed working memory, inhibition and planning, respectively. Children's vocabulary was tested using Peabody Picture Vocabulary Test, and included as control variables in addition to socioeconomic status of the participated families. The results showed that television viewing time and childdirected educational programs were positively associated with EF. In addition, television content fully mediated the effect of television viewing time on EF and parental restrictive approach strategies moderated the effect of television viewing time on EF.

Keywords: television exposure, executive function, parental mediation, preschoolers

\section{INTRODUCTION}

With the development and widespread use of electronic media, children now are using electronic media more and more, often at a very early age. Electronic media may be especially influential to children during periods of rapid brain development and plasticity (Christakis et al., 2012; Bush and Boyce, 2014) and may have powerful impacts on the development of social emotional competencies (e.g., Babaroglu, 2013) and cognitive capacities including executive function (EF) (e.g., Lillard and Peterson, 2011; Nathanson et al., 2014). EF refers to a family of top-down mental processes that aid in the monitoring and control of thought and action, and develops at a fast rate during the preschool period (Miller and Cohen, 2001). EF skills are essential for success in school and in life; and cognitive, social, and psychological development (Diamond, 2013). Many studies have addressed the possible effects of television exposure on children's EF (e.g., Barr et al., 2010; Wartella et al., 2010; Lillard and Peterson, 2011; Nathanson et al., 2014) because television is the most prominent media in the lives of young children. However, the results of these many studies have been contradictory: Some have reported negative effects of television viewing on children's 
EF (Barr et al., 2010; Lillard and Peterson, 2011; Nathanson et al., 2014), while others have found that children's television viewing facilitated their EF (Linebarger et al., 2014). It has recently been suggested that the potential effects of media use depend not only on what is watched but also how it is watched (McHale et al., 2009; Connell et al., 2015). In the context of children's media use, parental mediation behaviors regarding children's media use may play a critical role in moderating the effect of media use on children's EF.

While studies of EF development have focused on American or European children, few studies have examined the relation between television exposure and EF in other cultures, including Chinese culture. On the one hand, television programs in China and the amount of time that Chinese children spend watching television may be different from other cultures. In addition, unlike other western nations that provide guidelines about young children's screen media use, there are no official recommendations regarding children's digital media use or television viewing in China. On the other hand, Chinese children perform better than their western counterparts on EF tasks (Sabbagh et al., 2006). This may be partly because Chinese parents (Chao and Tseng, 2002) and teachers (Wang and Mao, 1996; Lan et al., 2009) request for self-control in everyday conduct. Investigating the relations between television exposure and the development of EF among Chinese preschoolers thus allows the examination of cultural similarities and differences in comparison with the results already reported in the literature.

Various theories have been proposed to explain why media exposure including television viewing may influence child development (Nikkelen et al., 2014). There are several potential ways that television exposure may impact child development outcomes. The first involves television watching time, which may displace activities such as reading or sleeping (Anderson et al., 2001). Still, previous research suggests that media use typically displaces functionally similar activities rather than activities that are more educationally valuable (Kirkorian and Anderson, 2009). The second way involves the content of television programs. Violent media may lead to ADHD-related behaviors (such as attention problems, hyperactivity, and impulsivity) by its effect on children's arousal system (Bushman and Huesmann, 2006) or by activate a violence script (Anderson and Bushman, 2001). Other researchers suggest that the fast-paced media, by the frequent use of cuts and edits, built the child an attentional style of scanning and shifting (Jensen et al., 1997) which may interfere with the development of attentional capacities in tasks that require sustained attention (Christakis, 2009). The third way is about children's media use context such as parental guidance behaviors which can moderate the effect of media use.

Christakis (2009) proposed a conceptual model (Figure 1) to understand the effect of television exposure on child development outcomes. This model, which can also help analyze and explain the effect of television exposure on EF, suggests that what children watch (content) and how they watch (context) as well as how much they watch are important factors influencing the nature of television exposure effects (Nikkelen et al., 2014). Most previous studies focused on the direct effect of television exposure time or viewing content on child development (e.g., Wright et al., 2001;

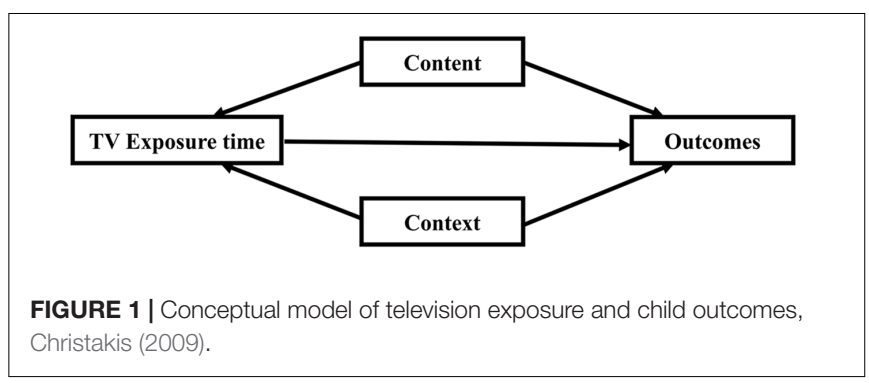

Nathanson et al., 2014). Recent study revealed television viewing context as important factors which can moderate the effect of television exposure (Linebarger et al., 2014). Based on the model posited by Christakis (2009), the current study was designed to examine the effect of three aspects of television exposure on Chinese preschoolers' EF: television viewing time, television content, and parental mediation behaviors on child's television viewing. We developed a moderated mediation model (Figure 2) by which we examined both the unique effects, namely, time and content, and the indirect effect (mediation effect) between these factors and children's EF, moderated by parent mediation behaviors impacting their children's television viewing to obtain a comprehensive understanding of the influence of television exposure. "A mediating variable transmits the effect of an independent variable on a dependent variable" (Mackinnon et al., 2007). Thus, the mediation analysis will help to identify potential processes between the independent variable and the dependent variable. Moderator is a variable that affects the strength of the relation between two variables. Moderator is usually an interaction, the relation between independent variable and dependent variable depends on a third variable (Mackinnon et al., 2007). Thus, the moderation analysis will help to answer how the relations between independent variable and dependent variable would change under different contexts. In addition, if a mediation effect depends on a third variable, the mediation effect was moderated and the moderated mediation analysis is needed. In other word, the mediation effect cannot remain constant across different contexts, groups of individuals, or values of the independent variable (Preacher et al., 2007).

Much research on the effect of television exposure has focused on attention, which is the vital foundation of EF processes (Posner and Rothbart, 2007). Yet these studies have reported mixed results (Christakis et al., 2004; Stevens and Mulsow, 2006; Foster and Watkins, 2010). Most of the published studies suggested that television viewing time is associated with lower attention skills (Landhuis et al., 2007; Swing et al., 2010). However, other studies revealed little effect of television viewing on attention problems (Stevens and Mulsow, 2006; Foster and Watkins, 2010). Similarly, research on relations between overall time spent viewing television and $\mathrm{EF}$ also showed mixed results: Nathanson et al. (2014) found that television viewing time was negatively related to EF, while Linebarger et al. (2014) found that low-risk preschoolers demonstrated higher EF with increased television viewing time. This inconsistency may be due to several issues. First, the relationship between television viewing time and EF was curvilinear, demonstrating that moderation level of 


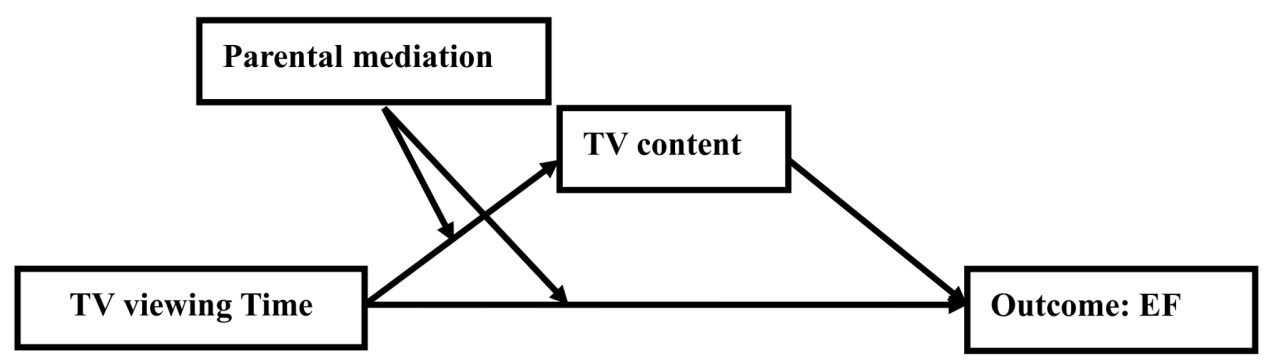

FIGURE 2 | The proposed conceptual model of effects of television exposure on EF.

viewing was positively associated with academic achievement, while additional viewing had an increasingly negative impact on achievement (Williams, 1982; Razel, 2001). Secondly, much of existing studies, a single indicator was used to measure television exposure without distinguishing between different types and dimensions of television program content.

Previous research has also indicated that both the age when children start watching television and television content matter. Numerous studies revealed educational programs such as Sesame Street appear to be beneficial for young viewers' language development and school readiness (e.g., Wright et al., 2001). Barr et al. (2010) found that children who watched higher amounts of adult-directed programs at age 1 showed poorer EF than those who started at age 4; in contrast, exposure to child-directed television programs at either age 1 or 4 was not associated with EF at age 4. Lillard and Peterson (2011) reported that fast-paced cartoons viewing would weaken the EF performance of 4 years old children. Their further research (Lillard et al., 2015a,b) revealed that fantastical content, not fast pacing, disrupted preschooler' EF. However, Linebarger et al. (2014) showed that low-risk preschoolers demonstrated better EF with increasing exposure to non-educational television (both child-directed entertainment and adult-directed content). On the other hand, Nathanson et al. (2014) found that PBS viewing was positively related to EF while educational cartoon viewing was related to poorer EF performance. The possible reason for this inconsistency in the relation between television content and EF may be the somewhat different criteria used to categorize television content. In Linebarger et al. (2014) study, they used an established protocol to code the reported programs. While, Nathanson et al. (2014) used principal component analysis to get the children's genre viewing. Additionally, the influence of television content and viewing time on young children's EF may moderated by other factors, such as the context of children's television viewing.

Parents play a crucial role in moderating the effects of television. Previous studies on parental mediation behaviors about children's media use (Valkenburg et al., 1999) have defined three distinct strategies: restrictive mediation, coviewing, and instructive mediation. Restrictive mediation includes parents' rules about the amount viewing time, permissible or forbidden types of content, and the use of viewing as a behavioral reward or punishment. Parent-child coviewing is defined as a shared set of motivations for viewing. Instructive mediation refers to an effort to discuss television content with children (Valkenburg et al., 1999). Studies have revealed that television co-use was associated with positive outcomes, including improves in comprehension and increased enjoyment of television content among preschoolers (Salomon, 1977), as well as increased attention and responsiveness among toddlers (Barr et al., 2008). In addition, parental mediation behaviors are associated with less children's television viewing time (Bulck and Bergh, 2000; An and Lee, 2010; Wu et al., 2014). Linebarger et al. (2014) found that parenting style moderated the relation between children's television exposure and EF. However, studies have not examined whether and how the associations between television exposure and children's EF may be moderated by parental mediation which was the more specific aspect of parenting style about children's media use. To address this issue, we tested whether the relation between television viewing and EF would be moderated by parental mediation.

In sum, the relationship between television viewing and EF is not well understood. To address this point, we conducted a comprehensive survey and laboratory measurement in 3- to 6-year-old children. Specifically, we aimed to examine how EF is associated with children's television viewing time (overall amount of television viewing) and viewing of different content (i.e., adult-oriented and child-oriented television), and to explore the moderating role of parental mediation in these relationships. When measuring television content, we differentiated between adult-directed content and child-directed content, and regarding child-directed content, we further differentiated different kinds of programs, such as educational content, recreational content, and violent content. Last and most important, we extended Christakis' (2009) model by proposing that television content would mediate the effect between television viewing time and EF. Given that viewing time always involves some type of content, the effect of viewing time would then be at least partly mediated by this content. The conceptual model of the present study is illustrated in Figure 2.

We formulated and tested the following hypotheses:

(1) Time spent television viewing will positively correlate with $\mathrm{EF}(\mathrm{H} 1)$;

(2) Television content will correlate with EF. Specifically, child-directed educational programs will positively correlate with EF (H2a) child-directed fast paced 
or violent programs (H2b) as well as adult-directed programs will negatively correlate with $\mathrm{EF}(\mathrm{H} 2 \mathrm{c})$;

(3) Television content will mediate the effect of television viewing time on $\mathrm{EF}(\mathrm{H} 3)$;

(4) Parental mediation will moderate the direct effect of television viewing time on EF ( $\mathrm{H} 4 \mathrm{a})$, and will moderate the indirect effect of television viewing time on EF through television content $(\mathrm{H} 4 \mathrm{~b})$.

\section{MATERIALS AND METHODS}

\section{Participants}

Participants were recruited from one preschool in a large city in northern China. A total of 119 preschoolers (51\% girls) age range from 3.17 to 6.31 years old $(M=4.64$ years old, $S D=0.92)$ and their parents $(73 \%$ mothers $)$ participated in this study. Participants are Han Chinese and speak Mandarin. We adopted two widely used markers of SES: maternal education and household income. $21.8 \%$ mothers held a graduate degree, $67.2 \%$ mothers were college educated, and $11 \%$ mothers indicated they had completed high school. Parents demonstrated their average monthly household income by choosing one of seven income levels, ranging from "less than RMB3,000" to "RMB30,000 or more." The largest percentage of participants selected "RMB3,000 to RMB6,000" (33.6\%), followed by "RMB6,000 to RMB8,000" (31.9\%), "RMB8,000 to RMB10,000" (16.8\%), "RMB10,000 to RMB15,000" (11.8\%), "RMB15,000 to RMB30,000" (2.5\%), and "less than RMB 3,000" (3.4\%). The average annual household income of Chinese was 49,318 RMB in 2014 (He, 2015). Most families' household incomes in the present study were above the national average income. Most of the families (79\%) owned one television, and some of the families (18\%) had more than two televisions, however, none placed televisions in children's bedrooms.

\section{Procedure}

This study was carried out in accordance with the ethical standards of Declaration of Helsinki and was approved by the Research Ethics Board of the Institute of Psychology, Chinese Academy of Sciences. Children were recruited using simple random sampling method based on the name list provided by the preschool. Parents were given consent forms and finished a questionnaire including their demographics and the television use of the participating child as well as parental guidance patterns on children's television use. Children were interviewed one by one in a quiet room at their preschool by three trained researchers. The experimenters administered EF tasks and a vocabulary assessment during three sessions, each lasting between 10 and 20 min to avoid that children cannot concentrate on the tasks. All three sessions were done in the same day. The tasks in each session were in a fixed order. The order in which children completed the three test sessions was randomly assigned to eliminate possible order effects. Session 1 included the backward digit span task, the spatial span task and Peabody picture vocabulary test. Session 2 included the boy-girl Stroop, Simon task, and flanker task. Session 3 included the tower of
Hanoi task. We gave each participated child a small gift and each parent some gifts for their participation.

\section{Children's Television Exposure Measures Television Viewing by Children}

Parents estimated the hours their child watched television on an average weekday and on an average weekend day during three time periods, namely, morning, midday, and evening, respectively. Parents were told that viewing estimates should include "programs seen on a television, computer, or via DVD or a portable electronic device.” (Nathanson et al., 2014) The sum for weekday viewing was then multiplied by 5 and the sum for weekend viewing was multiplied by 2 . We then summed the two products and divided it with 7 to generate average daily viewing in hours $(M=1.22 \mathrm{~h}, S D=0.93)$.

\section{Age of Starting to Watch Television}

Parents reported the age at which their child first began watching television (Nathanson et al., 2014), choosing their answer on a scale from "never done," "before 6 months old," "between 6 months old and 1 year old," "1 year old," "2 years old," "3 years old," "4 years old" to "at or after 5 years old."

\section{Children's Channel Viewing}

In China, China Central Television (CCTV) and local television stations both have special channels to broadcast children's programs. The highest ratings and the most popular children's channels include CCTV-14 Children's Channel, Golden Eagle Cartoon Channel of Hunan TV, and KAKU Children's Channel of Beijing TV. On a scale from 0 (never) to 3 (all of the time), parents reported how often their child watched six different channels when they watched television, including CCTV-14 Children's Channel, Golden Eagle Cartoon Channel, KAKU Children's Channel, other cartoon channels, CCTV (other than the children's channel), and other channels. A principalcomponents factor analysis with oblimin rotation was used to explore the factors of children's channel viewing. Two factors were extracted, explaining approximately $58.24 \%$ of variance. The first factor consisted of the viewing of CCTV-14 Children's Channel, KAKU Children's Channel, Golden Eagle Cartoon Channel, and other cartoons channels, explaining about $35.5 \%$ of the variance. The second factor included viewing of CCTV (other than the children's channel) and other channels, and explained $22.75 \%$ of the variance. Two factors were constructed into two scales to represent child-directed programs viewing and adultdirected programs viewing. The Cronbach's alpha coefficients of the two scales were 0.70 and 0.65 .

\section{Children's Genre Viewing}

Based on the categories used in a previous study (Nathanson et al., 2014), parents reported how often their child watched various types of entertainment or educational content when they watched television. Using a scale from 0 (never) to 3 (all of the time), these program categories included "action cartoons (e.g., Kung Fu Panda, Ultraman)," "classic cartoons (e.g., Tom and Jerry)," "live educational children's programs (e.g., Tree of Knowledge)," "fast-paced cartoons (e.g., Phineas and Ferb, 
Sponge Bob Square Pants)," "situation comedies for children (e.g., Star Elves Lan Duoduo, Balla Balla Little Magic Fairies)," and "educational cartoons (e.g., Dora the Explorer, Rainbow Cat, Blue Rabbit ).”

\section{Parental Mediation Behaviors on Children's Television Use (Bulck and Bergh, 2000)}

Parents were asked to indicate their mediation behavior regarding to children's television viewing. They were asked to choose one of four answers (never, seldom, sometimes, and often) to each of 12 questions representing three types of parental mediation approaches. The three types of parental approaches included: (a) Instructive approach (e.g., "Tell my child when somebody in a show does something that is bad"); (b) Coviewing approach (e.g., "Watching television together with my child because it is important to her or him to do this activity together with me"); and (c) Restrictive approach (e.g., "Not allow my child to watch a certain program"). In this study, the Cronbach's alpha coefficient of the three sub-scales were $0.80,0.69$, and 0.68 .

\section{Child Measures of EF}

Executive functions tasks were selected based on evidence for their reliability and appropriateness for use with preschoolers. EF was viewed as a set of complex cognitive skills involved in controlling, directing, and planning cognitive activities and behaviors (Diamond, 2013). In the present study, children's working memory, inhibition, and planning were measured to represent the different aspects of EF development. In addition, these EF components are highly correlated and are essentially a single component during preschool years (Wiebe et al., 2008) and aggregated measures of EF are stable and robust (Willoughby et al., 2011). Based on previous study (Nathanson et al., 2014), scores on all the measured EF tasks were standardized to $Z$-scores and then summed to create an overall assessment of children's EF. Higher scores of EF reflect better abilities of EF.

\section{Working Memory}

The backward digit span task (Carlson et al., 2002) and the spatial span task were used to test children's working memory. In the backward digit span task, the child was told a series of digits and asked to repeat the digits in reverse. Children received three trials each of two-, three-, and four-digit lengths. The tasks were ended when children gave wrong answers on two trials of a given length. Performance was measured with the highest digit- length completed. In the spatial span task, a little chicken appeared on the computer screen at different locations in a $3 \times 3$ grid, with each appearance remaining on the screen for $1000 \mathrm{~ms}$. Children were instructed to reproduce the sequence of the locations by touching the grids. There were three trails in each level. The task was ended if children failed more than twice on one given level. Performance was measured with the highest level completed.

\section{Inhibition}

Three tasks were used to assess different aspects of inhibition. In Boy-Girl Stroop task (Miller et al., 2012) children were instructed to say "boy" when they see a cartoon girl on the screen and to say "girl" when seeing a cartoon boy on the screen. The task included
20 pictures, with each type of picture appearing 10 times. Performance was measured with the number of times children correctly labeled each picture. In the Simon task (Davidson et al., 2006), a color picture of either a frog or butterfly was presented on the left or right side of the computer screen. The appearance of frog was always associated with right key press response and the butterfly was associated with left key press response. The stimuli were presented randomly on the left or right of the screen over the block of 20 trials, yielding congruent and incongruent trials. In the Flanker task (Fan et al., 2002), children were presented with a row of five fish with the target fish flanked on each side by two fish facing the same or the opposite direction (congruent or incongruent conditions, respectively). Children need to identify, the direction the target fish was facing on each trail by key press. There were two blocks each consisted of 20 trials and the target stimuli were presented randomly facing left or right.

\section{Planning}

The Tower of Hanoi task (ToH) (Lillard and Peterson, 2011) involved a base with three long pegs and a larger and a smaller disk that fit on the pegs, and a picture portrayal a goal state. A story about helping monkeys home was introduced to help the child to understand the rules of this task: only 1 disk could be moved at a time, the disks always needed to stay on pegs, and the bigger disk could never go on top of the smaller disk. The children were given a score of 1 if they moved the disks successfully by following all the rules. Children who broke a rule or failed to complete the task were given a score of 0 .

\section{Covariates}

Children's age, gender and family SES have been linked to differences in the amount and quality of television exposure (Anderson et al., 2001; Zimmerman and Christakis, 2007) as well as the development of EF (Linebarger et al., 2014) were as covariates in all models. Children's vocabularies were also controlled as covariate because children with higher language ability may perform better on EF tasks due to their better understanding the task instructions (Bernier et al., 2010). Children's receptive vocabulary was assessed with the Peabody picture vocabulary test - Chinese edition (PPVT-C) (Sang and Miao, 1990). The experimenter stated a word, and children had to point to the corresponding picture out of four choices. The task ended when children made more than five errors on a set of eight words. Performance was measured in terms of raw scores (i.e., ceiling item - total errors).

\section{Analysis Plan}

Multiple hierarchical linear regression models were computed with SPSS 19.0 to test the direct effect of television viewing time, viewing content and parental mediation on EF (Hypotheses 1 and 2). SPSS macro (PROCESS; Preacher et al., 2007; Hayes, 2015) was run to test the moderated mediation model (Hypotheses 4). PROCESS is a bootstrapping procedure, which provide the estimates of the confidence intervals (CIs) of the indirect effect or conditional indirect effect from bootstrapped samples. Conditional indirect effect is the magnitude of an indirect effect at a particular value of the moderator 
(Preacher et al., 2007). If the conditional indirect effects at levels moderator were significantly different, then the mediation effect was moderated. In order to test the significance of the moderated mediation effect, Hayes (2015) proposed a term called index of moderated mediation. The index of moderated mediation is a quantification effect of the moderator on the indirect effect of independent variable on dependent variable through the mediator (Hayes, 2015). If the 95\% CI of the index did not contain zero, the moderated mediation was significant. A bootstrapping approach provides much greater statistical power for mediation analysis than the traditional causal steps approach (Hayes, 2009). Variance inflation factors (VIF) was used to diagnose multicollinearity of these models. Due to the non-normal distributions of children's television viewing time ( skewness $=1.88$, kurtosis $=6.29$ ), a square-root transformation was conducted to make the distributions more normal. This strategy was used by previous researches to deal with the media use variables (e.g., Ferguson and Brent, 2014).

\section{RESULTS}

The descriptive statistics for all the measures are displayed in Table 1.

Table 2 presents the bivariate correlations between non-media variables, media exposure variables and EF. Children's EF was highly positively correlated with their age and vocabulary. Positive correlations were evident among television viewing time, children's channels and cartoon programs. Moreover, the television viewing time was not significantly correlated with EF. While, child's channels viewing and certain types of cartoon programs including classic cartoons viewing, fast-paced cartoons viewing, and children's situation comedies viewing were positively correlated with EF. These zero-order correlations should be interpreted with caution. For instance, older children may watch more television and have better EF development than younger children. The results indeed revealed that child's age was positively related with child's channels viewing and certain types of cartoon programs. Thus, further regression analysis with these confounding variables under control was conducted.

Four separate hierarchical regression models for each set of media exposure variables were conducted to test hypotheses 1 and 2: the direct effect of television viewing time, viewing content and parental mediation (Table 3). In each model child's age, gender, vocabulary, maternal education and family income were as control variables entered in first step. Children's television viewing time and the age start watching television, children's channel viewing, children's genre viewing and parental guidance patterns on children's television use, were entered in second steps of Model 1 Modle 4 separately. These models allowed us to observe the unique contribution of each set of media variables to the variance of EF above and beyond the control variables. VIFs of the four models ranged from 1.03 to 2.60, which were lower than the recommended level (VIF < 10) (Hair et al., 1995) suggesting that these models did not violate the stability of the parameter estimates. Across the four models, child's age, gender, vocabulary, maternal education, and family income accounted for a significant $67 \%$ of the variance in $\mathrm{EF}$ with child's age $(\beta=0.55, p<0.001)$, vocabulary $(\beta=0.33$, $p<0.001)$ and mother education $(\beta=-0.12, p<0.05)$ as significant predictors. In Model 1, Children's television viewing time and the age start watching television explained a significant $2 \%$ of additional variance with television viewing time related to better EF, Cohen $f^{2}=0.06$. In Model 2, children's channel viewing did not account for significant variance in EF, Cohen $f^{2}=0.01$. In Model 3, six kinds of children's cartoons or programs explained a significant $7 \%$ of additional variance in EF, Cohen $f^{2}=0.27$. Classic cartoons viewing was significantly positively related with EF. Live educational children's programs viewing was marginal significantly positively related with $\mathrm{EF}(\beta=0.12$, $p=0.065)$. While, educational cartoons viewing was marginal significantly negatively related with $\mathrm{EF}(\beta=-0.13, p=0.056)$. In Model 4, three kinds of parental guidance patterns account for a significant $3 \%$ of additional variance in $\mathrm{EF}$ with restrictive approach significantly related with poor EF, Cohen $f^{2}=0.1$. Cohen's categories about effect size (Cohen, 1977) indicates that effect size of $f^{2}$ smaller than 0.02 is small effect, 0.15 is moderate effect and 0.35 means large effect. The effect size of television

TABLE 1 | Descriptive analysis of the variables.

\begin{tabular}{|c|c|c|c|c|}
\hline Variables & $M$ & $S D$ & Min & Max \\
\hline Child's age (years) & 4.64 & 0.92 & 3.17 & 6.31 \\
\hline Maternal education & 3.11 & 0.56 & 2 & 4 \\
\hline Family income & 3.08 & 1.16 & 1 & 6 \\
\hline Child vocabulary & 59.17 & 31.27 & 9 & 134 \\
\hline TV viewing (hours) & 1.22 & 0.93 & 0 & 6.14 \\
\hline Onset of TV viewing (years) & 1.36 & 0.80 & 0.5 & 4.0 \\
\hline Child channels viewing & 1.39 & 0.71 & 0 & 3 \\
\hline Adult channels viewing & 0.51 & 0.46 & 0 & 2 \\
\hline Action cartoons viewing & 0.81 & 0.78 & 0 & 3 \\
\hline Classic cartoons viewing & 1.35 & 0.70 & 0 & 3 \\
\hline Children's live show programs viewing & 1.19 & 0.83 & 0 & 3 \\
\hline Fast-paced cartoons viewing & 1.25 & 0.82 & 0 & 3 \\
\hline Educational cartoons viewing & 1.29 & 0.93 & 0 & 3 \\
\hline Children's situation comedies viewing & 0.53 & 0.72 & 0 & 3 \\
\hline Restrictive approach & 3.23 & 0.65 & 1 & 4 \\
\hline Coviewing approach & 3.23 & 0.58 & 1 & 4 \\
\hline Instructive approach & 3.31 & 0.65 & 1 & 4 \\
\hline Back-digit span & 1.84 & 0.83 & 1 & 5 \\
\hline Spatial span task & 3.12 & 1.12 & 1 & 6 \\
\hline Boy-girl stroop & 10.47 & 5.83 & 0 & 20 \\
\hline Tower of Hanoi & 0.36 & 0.53 & 0 & 2 \\
\hline FL-in- acc & 0.72 & 0.26 & 0.25 & 1.00 \\
\hline SI-in-acc & 0.83 & 0.17 & 0.25 & 1.00 \\
\hline EF & 0 & 4.30 & -9.83 & 9.31 \\
\hline
\end{tabular}

Categories for parent education were 1 = less than junior high school, 2 = high school, 3 = undergraduate, 4 = graduate degree; Categories for family income 1 = less than 3,000 Yuan, $2=3,000 \sim 6,000$ Yuan, $3=6,000 \sim 8,000$ Yuan, $4=8,000 \sim 10,000$ Yuan, $5=10,000 \sim 15,000$ Yuan, $6=15,000 \sim 30,000$ Yuan, $7=$ more than 30,000 Yuan. FL-in-acc = the accuracy of incongruent trials in Flanker task; SI-in-acc = the accuracy of incongruent trials in Simon task; EF was the composite Z-scores of six tasks. 


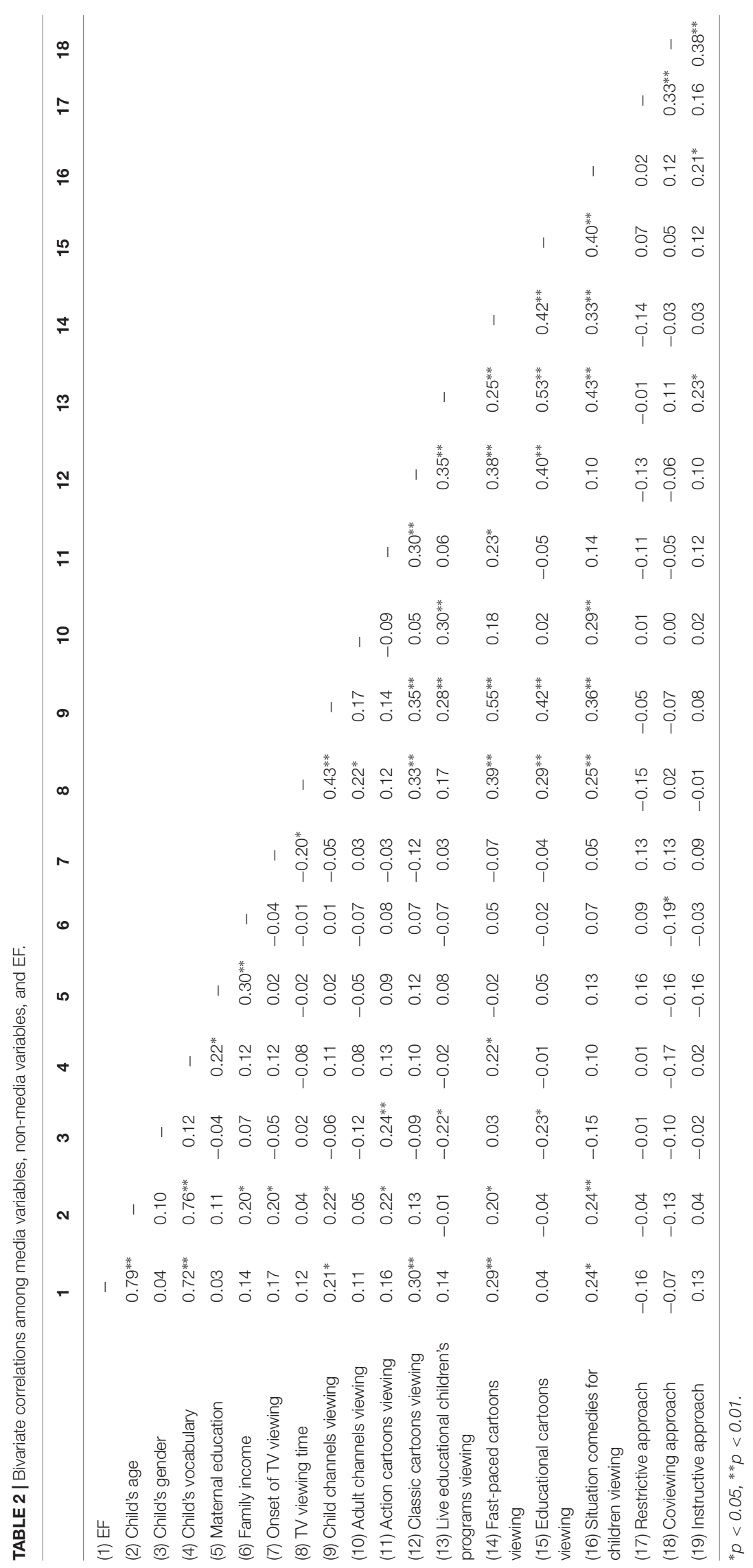


viewing time account for EF was small to medium effect and the effect size of viewing content was medium to large effect.

SPSS macro PROCESS (Model 4) (Hayes, 2015) was run to test the multiple mediator model for Hypotheses 3. The results showed that the only indirect effect estimate for television viewing time on $\mathrm{EF}$ through classic cartoons viewing $(a b=0.24$, $S E=0.11,95 \%$ bias corrected CI $[0.08,0.53])$ and live educational children's programs viewing $(a b=0.10, S E=0.06,95 \%$ bias corrected CI $[0.01,0.29])$ were significant because both 95\% bias corrected CI did not contain zero. In each analysis, maternal education and family income, children's age, gender, and vocabulary were controlled. The mediated model explained $69 \%$ variance in $\mathrm{EF}(p<0.001)$. Number of bootstrap samples was 5000 .

We further applied the SPSS macro PROCESS (Model 8) (Hayes, 2015) to test if restrictive approach strategies can moderate the mediation model (Hypotheses 4). In each analysis, we controlled for maternal education and family income, children's age, gender, and vocabulary. Number of bootstrap samples was 5000. Conditional direct effects and indirect effects of television viewing time on EF were tested at three levels of restrictive approach: the mean level of restrictive approach and one SD below and above the mean level. Result showed that firstly, restrictive approach strategies moderate the direct effect of television viewing time on EF. Television viewing time had a positive effect on EF only at the low level of restrictive approach $(B=0.76, S E=0.33,95 \%$ CI $[0.10,1.42])$. Secondly, restrictive approach strategies moderated the indirect effect of television viewing time on EF through child classic cartoons viewing, with the index of moderated mediation equal to -0.17 $(S E=0.12,95 \%$ CI $[-0.47,-0.003])$. The index was negative, meaning that the indirect effect of television viewing time on EF through child classic cartoons viewing was a decreasing function of restrictive approach strategies. Conditional indirect effect(s) of television viewing time on EF at values of the restrictive approach showed that only at a low to modest level of restrictive approach, there was a positive indirect effect of television viewing time on EF through child classic cartoons viewing $(a b=0.28, S E=0.16,95 \%$ bias corrected CI $[0.05$, $0.67]$ and $a b=0.17, S E=0.08,95 \%$ bias corrected CI [0.03, $0.42]$ ). Thirdly, restrictive approach strategies did not moderate the indirect effect of television viewing time on EF through live educational children's programs viewing, with the index of moderated mediation equal to -0.01 ( $S E=0.07,95 \% \mathrm{CI}$ $[-0.19,0.11]), 95 \%$ CI of the indexes contain zero, suggesting that the tests of moderated mediation were not statistically significant. However, the indirect effect of television viewing time on EF through live educational children's programs viewing was significant only at the modest level of restrictive approach $(a b=0.08, S E=0.05,95 \%$ bias corrected CI $[0.01,0.23])$. Results about the moderated mediation model was presented in Table 4 and Figure 3.

\section{DISCUSSION}

The present study is one of few studies conducted to examine the relations between television viewing and cognitive outcomes among Chinese preschoolers. Most importantly, the study extends earlier work by considering multiple variables related to children's television viewing behavior: how old they were when they started watching, how much time they watch (television viewing time), what they watch (television viewing content), and how they watch (television viewing context: parental behaviors related to children's television viewing), as well as multiple measures of EF. Results of the study yielded significant implications for understanding what, how, and when television viewing affects the development of EF. In addition, the present results also suggest both cultural similarities and differences in the associated patterns reported in the literature.

The result that television viewing time was positively associated with EF confirms Hypothesis 1. Hypothesis 2 was also supported by the finding that although child-directed live educational television and classical cartoons viewing was

TABLE 3 | Summary of hierarchical regression analyses for non-media and media variables predicting children's EF.

\begin{tabular}{|c|c|c|c|c|c|c|c|}
\hline & Variables & $B$ & SE & $\beta$ & Unique $R^{2}$ & $F$ change & Cohen $f^{2}$ \\
\hline \multirow[t]{2}{*}{ Model 1} & Onset of TV viewing & 0.31 & 0.30 & 0.06 & 0.02 & $3.60^{*}$ & 0.06 \\
\hline & TV viewing time & 0.58 & 0.22 & $0.15^{* *}$ & & & \\
\hline \multirow[t]{2}{*}{ Model 2} & Child channels viewing & 0.27 & 0.34 & 0.05 & 0.004 & 0.708 & 0.01 \\
\hline & Adult channels viewing & 0.40 & 0.52 & 0.04 & & & \\
\hline \multirow[t]{6}{*}{ Model 3} & Action cartoons viewing & -0.49 & 0.32 & -0.09 & 0.07 & $4.42^{* * *}$ & 0.27 \\
\hline & Classic cartoons viewing & 1.44 & 0.39 & $0.23^{* * *}$ & & & \\
\hline & Live educational children's programs viewing & 0.62 & 0.33 & $0.12^{\mathrm{a}}$ & & & \\
\hline & Fast-paced cartoons viewing & 0.23 & 0.32 & 0.04 & & & \\
\hline & Educational cartoons viewing & -0.61 & 0.32 & $-0.13^{b}$ & & & \\
\hline & Situation comedies for children viewing & 0.42 & 0.38 & 0.07 & & & \\
\hline \multirow[t]{3}{*}{ Model 4} & Restrictive approach & -1.16 & 0.38 & $-0.18^{* *}$ & 0.03 & $3.85^{*}$ & 0.1 \\
\hline & Co-using approach & 0.59 & 0.47 & 0.08 & & & \\
\hline & Instructive approach & 0.55 & 0.38 & 0.08 & & & \\
\hline
\end{tabular}

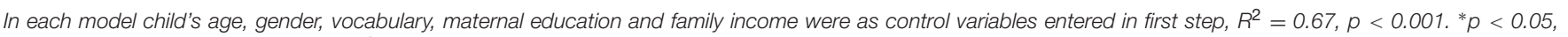
${ }^{* *} p<0.01,{ }^{* * *} p<0.001,{ }^{a} p=0.065,{ }^{b} p=0.056$. 
TABLE 4 | Summary of the moderated mediation model.

\begin{tabular}{|c|c|c|c|c|c|c|c|c|c|}
\hline \multirow[b]{2}{*}{ Variables } & \multicolumn{3}{|c|}{$\begin{array}{c}\text { Mediator: Classic cartoons } \\
\text { viewing }\end{array}$} & \multicolumn{3}{|c|}{$\begin{array}{l}\text { Mediator: Live educational } \\
\text { children's programs viewing }\end{array}$} & \multicolumn{3}{|c|}{ Outcomes: EF } \\
\hline & $B$ & $S E$ & $\beta$ & $B$ & SE & $\beta$ & $B$ & $S E$ & $\beta$ \\
\hline Age & 0.02 & 0.11 & 0.02 & 0.00 & 0.13 & 0.00 & 2.39 & 0.37 & $0.51^{* * *}$ \\
\hline Gender & -0.18 & 0.12 & -0.13 & -0.35 & 0.15 & $-0.22^{*}$ & -0.35 & 0.44 & -0.04 \\
\hline Child's vocabulary & 0.00 & 0.00 & 0.08 & 0.00 & 0.00 & 0.01 & 0.05 & 0.01 & $0.34^{* * *}$ \\
\hline Maternal education & 0.13 & 0.12 & 0.11 & 0.15 & 0.15 & 0.10 & -0.94 & 0.41 & $-0.12^{*}$ \\
\hline Family income & 0.02 & 0.06 & 0.03 & -0.06 & 0.07 & -0.09 & 0.14 & 0.20 & 0.04 \\
\hline TV viewing time & 0.22 & 0.06 & $0.35^{* * *}$ & 0.14 & 0.07 & $0.18^{*}$ & 0.27 & 0.21 & 0.07 \\
\hline Restrictive approach & -0.13 & 0.10 & -0.12 & 0.00 & 0.12 & 0.00 & -0.78 & 0.34 & $-0.12^{*}$ \\
\hline Classic cartoons viewing & & & & & & & 0.75 & 0.35 & $0.12^{*}$ \\
\hline Children's live educational programs viewing & & & & & & & 0.55 & 0.28 & $0.11 *$ \\
\hline TV viewing time $\times$ Restrictive approach & -0.22 & 0.10 & $-0.20^{*}$ & -0.02 & 0.12 & -0.02 & -0.75 & 0.35 & $-0.11^{*}$ \\
\hline$R^{2}$ & $0.20^{* *}$ & & & 0.09 & & & $0.75^{* * *}$ & & \\
\hline
\end{tabular}

Restrictive approach and television viewing time were mean centered. ${ }^{*} p<0.05,{ }^{* *} p<0.01,{ }^{* * *} p<0.001$.

positively associated with EF while adult-directed television and other kinds programs were not significantly correlated with EF. In addition, it is evident that child's live educational program and classical cartoons viewing mediated the effect of television viewing time on EF, and these results confirm Hypothesis 3. Parental restrictive approach strategies were also found to moderate the direct effect of television viewing time on EF, as well as the indirect effect of television viewing time on EF through child classic cartoons. Hypothesis 4 which indicated that parental mediation can moderate the direct effect of television viewing on $\mathrm{EF}$ and can moderate the indirect effect of television viewing on EF through television content were confirmed. Our proposed conceptual model (Figure 2) was thus confirmed.

The positive association between television viewing time and EF evident in the present study is different from most previous studies, which showed negative or null associations between television viewing time and EF (Nathanson et al., 2014; Blankson et al., 2015). These inconsistencies in the nature of the relation between television viewing and EF may be explained with the following possibilities.

First, the relation between television viewing time and the development of EF appears to be a reverse $U$ shape, instead of a linear function (Hofferth, 2010). A significant difference between the current and previous results is that the television viewing time of this Chinese sample was significantly shorter than their counterparts reported in other studies $(M=1.22 \mathrm{~h}$, $S D=0.93$ vs. $M=2.87 \mathrm{~h}, S D=1.99$ ) (Nathanson et al., 2014). Other previous investigation about Chinese preschooler's media use showed similarity result that they spent about 90 min per day to watch television (Li et al., 2014). Foster and Watkins (2010) also suggested that the non-linear specification reveals an association between television watching and attention problems that exists only at very high levels of television viewing. Similarly, several other studies (Obel et al., 2004;

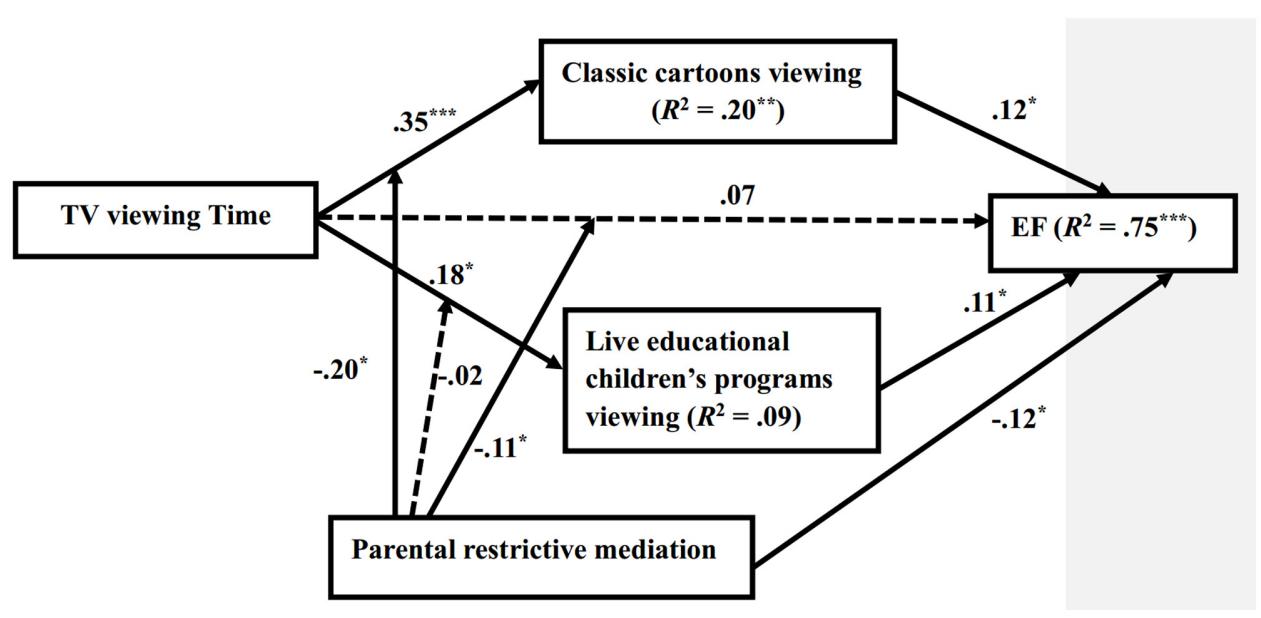

FIGURE 3 | Summary of the moderated mediation model. Restrictive approach and television viewing time were mean centered. Child's age, gender, vocabulary, maternal education, and family income were as control variables. ${ }^{*} p<0.05,{ }^{* *} p<0.01,{ }^{* * *} p<0.001$. Dotted lines indicate the coefficients were not significant. 
Christakis, 2009) have shown a null relationship between television viewing and attention problems in low levels of viewing.

Second, what children watch (content) and how they watch (context) are arguably more important television viewing variables than how much children watch. As revealed in the present study, the effect of television viewing time on EF disappeared when television viewing content was included in the regression model, suggesting that television viewing content fully mediated the effect of television viewing time on EF. It is revealed that classical cartoons and live educational programs viewing were positively correlated with EF. There are many live educational programs such as tree of knowledge directed to preschoolers. These high-quality programs may enable children to develop EF skills.

Third, it is important to consider children's media use context as the moderate effect of parental restrictive behaviors was evident in the present study. The design of our study enabled us to examine the relation between television viewing and EF while taking context variables into consideration, and our findings suggest a more complex picture: the relation patterns between television viewing and EF also depend on parental restrictive behaviors. Restrictive mediation includes parents' rules regarding the amount of viewing, permissible and forbidden types of content, and the use of viewing as a behavioral reward or punishment. A significant positive relation between television viewing time and $\mathrm{EF}$ was evident only when restrictive mediation was at a low level. In addition, only when restrictive approaches were at low or moderate levels, there was a positive indirect effect of television viewing time on EF through child classical cartoon and live educational programs viewing. Combined with the results of bivariate correlation analyses, it is evident that a restrictive approach was negatively correlated with television viewing time and most kinds of child programs. Although the correlations were not significant, they suggest that parental restrictive behaviors tend to be associated with less television viewing.

A particularly interesting finding of this study was the main negative effect of parental restrictive approach strategies on EF. There are several possible explanations. A parental restrictive approach is associated with an arbitrary parenting style, which involves depriving children's opportunities to regulate their own behavior and requiring them to adapt continually to another's perspective (Talwar et al., 2011). Such an approach might be the cause of slow development of EF. An alternative explanation is that parents whose child demonstrates poor EF may set more limitations on their child's television watching. The present study urges parents and educators to be aware of the potential moderation effect of media use guidance behaviors on children's media use as well as their development. Parents should care about their arbitrary guidance behaviors about their children's media use. Future work is needed to further explore the role of parental meditation behaviors related to children's television viewing and its impact on EF.

Although not part of our major concern of the study, an unexpected result involving the relation between mother education and child's EF. Contrary to prior study (Sarsour et al.,
2011), we found mother education was negatively related to EF. This may partly due to the narrow range of mother education in this sample with most mothers hold university or college degree $(67 \%)$ and postgraduate degree (22\%).

In sum, the present study was conducted to examine the relations between children's television viewing and the development of EF with various features and strengths. The quality of the measures is one advantage. Our EF tasks were measured directly by children's responses instead of using parent report. Moreover, in the present study, we controlled other factors that could influence both children's EF and television viewing. Finally, our research was guided by a theoretical framework and the results largely confirmed the proposed conceptual model. The findings of this study suggest that EF is associated with differences in both television viewing time and content. These findings form an important first step toward the conceptualization and investigation of more nuanced models on the relationship between television viewing and EF. Future research should not only distinguish between the different types of content that children are exposed to, but must also more systematically conceptualize and model mediating and moderating factors in the investigation of relations between EF and television. Building such indirect and conditional effects models will enable us to gain a deeper understanding of the relationship between media use and EF.

Although our findings contribute to our understanding of the relation between young children's television viewing and the development of EF, there are still limitations in our study. First, as with most previous studies, the cross-sectional nature of this study prevents us from drawing conclusions about the direction and causality of the current results. Although television viewing might well affect the development of EF, we cannot exclude the possibility that children with higher EF ability are more capable of choosing child directed educational television programs and spend more time watching them (Wright et al., 2001). Research that further explores the bidirectional relationships between EF and television viewing is therefore urgently needed. Another major limitation of the present study is that the sample overall watched relatively little television. Further study with the nationally representative sample to examine the relations between television viewing and child cognition development is needed. Finally, the present study mainly focused on the effect of television exposure. Given the rapid rise in the use of mobile technology by young children, future studies should consider addressing issues related to relations between children's mobile media use and higher-order cognitive abilities.

\section{CONCLUSION}

This study reveals that television exposure is related to children's EF. Furthermore, the relation between television viewing and EF development depend on programming content and parents' media monitoring behaviors. Given the important role of EF in child's social function development and school achievement, as well as the widely use of electronic media by young children, increasing our understanding of the links between children's 
television viewing as well as the new interactive media and EF is urgently needed.

\section{AUTHOR CONTRIBUTIONS}

$\mathrm{XY}$ designed the study, collected and analyzed data, and drafted the paper. LZ co-designed and revised the paper critically. ZC and ZW revised the paper critically.

\section{REFERENCES}

An, S.-K., and Lee, D. (2010). An integrated model of parental mediation: the effect of family communication on children's perception of television reality and negative viewing effects. Asian J. Commun. 20, 389-403. doi: 10.1080/01292986. 2010.496864

Anderson, C. A., and Bushman, B. J. (2001). Effects of violent video games on aggressive behavior, aggressive cognition, aggressive affect, physiological arousal, and prosocial behavior: a meta-analytic review of the scientific literature. Psychol. Sci. 12, 353-359. doi: 10.1111/1467-9280.00366

Anderson, D. R., Huston, A. C., Schmitt, K. L., Linebarger, D. L., Wright, J. C., and Larson, R. (2001). Early childhood television viewing and adolescent behavior: the recontact study. Monogr. Soc. Res. Child Dev. 66, I-VIII, 1-147. doi: $10.2307 / 1129224$

Babaroglu, A. (2013). The Influence of TV to preschool children social relations and parents approaches to this subject. J. Psychol. Educ. Res. 21, 7-28.

Barr, R., Lauricella, A., Zach, E., and Calvert, S. L. (2010). Infant and early childhood exposure to adult-directed and child-directed television programming: relations with cognitive skills at age four. Merrill Palmer Q. 56, 21-48. doi: 10.1353/mpq.0.0038

Barr, R., Zack, E., Garcia, A., and Muentener, P. (2008). Infants' attention and responsiveness to television increases with prior exposure and parental interaction. Infancy 13, 30-56. doi: 10.1080/15250000701779378

Bernier, A., Carlson, S. M., and Whipple, N. (2010). From external regulation to self-regulation: early parenting precursors of young children's executive functioning. Child Dev. 81, 326-339. doi: 10.1111/j.1467-8624.2009.01397.x

Blankson, A. N., O’Brien, M., Leerkes, E. M., Calkins, S. D., and Marcovitch, S. (2015). Do hours spent viewing television at ages 3 and 4 predict vocabulary and executive functioning at age 5? Merrill Palmer Q. 61, 264-289. doi: 10.13110/ merrpalmquar1982.61.2.0264

Bulck, J. V. D., and Bergh, B. V. D. (2000). The influence of perceived parental guidance patterns on children's media use: gender differences and media displacement. J. Broadcast. Electron. Med. 44, 329-348. doi: 10.1207/ s15506878jobem4403_1

Bush, N. R., and Boyce, W. T. (2014). "The contributions of early experience to biological development and sensitivity to context," in Handbook of Developmental Psychopathology, M. Lewis and K. Rudolph (Berlin: Springer), 287-309.

Bushman, B. J., and Huesmann, L. R. (2006). Short-term and long-term effects of violent media on aggression in children and adults. Arch. Pediatr. Adolesc. Med. 160, 348-352. doi: 10.1001/archpedi.160.4.348

Carlson, S. M., Moses, L. J., and Breton, C. (2002). How specific is the relation between executive function and theory of mind? Contributions of inhibitory control and working memory. Infant Child Dev. 11, 73-92. doi: 10.1002/icd.298

Chao, R., and Tseng, V. (2002). "Parenting of Asians," in Handbook of Parenting: Social Conditions and Applied Parenting, 2nd Edn, Vol. 4, ed. M. Bornstein (Mahwah, NJ: Erlbaum), 59-93.

Christakis, D. (2009). The effects of infant media usage: What do we know and what should we learn? Acta Paediatr. 98, 8-16. doi: 10.1111/j.1651-2227.2008.01027.x

Christakis, D., Ramirez, J., and Ramirez, J. (2012). Overstimulation of newborn mice leads to behavioral differences and deficits in cognitive performance. Sci. Rep. 2:546. doi: 10.1038/srep00546

Christakis, D. A., Zimmerman, F. J., DiGiuseppe, D. L., and McCarty, C. A. (2004). Early television exposure and subsequent attentional problems in children. Pediatrics 113, 708-713. doi: 10.1542/peds.113.4.708

\section{FUNDING}

This work was supported by the National Social Science Foundation of China (14ZDB161), the Chinese Academy of Sciences (KJZD-EW-L04), the National Social Science Foundation of China for Young Scholars in Education (CBA150155), the Fundamental Research Funds for the Central Universities (GK201603127) and China Postdoctoral Science Foundation funded project (2016M602753).

Cohen, J. (1977). Statistical Power Analysis for the Behavioral Sciences. New York, NY: Academic Press.

Connell, S. L., Lauricella, A. R., and Wartella, E. (2015). Parental co-use of media technology with their young children in the USA. J. Child. Med. 9, 5-21. doi: 10.1080/17482798.2015.997440

Davidson, M. C., Amso, D., Anderson, L. C., and Diamond, A. (2006). Development of cognitive control and executive functions from 4 to 13 years: evidence from manipulations of memory, inhibition, and task switching. Neuropsychologia 44, 2037-2078. doi: 10.1016/j.neuropsychologia.2006.02.006

Diamond, A. (2013). Executive functions. Annu. Rev. Psychol. 64, 135-168. doi: 10.1146/annurev-psych-113011-143750

Fan, J., McCandliss, B. D., Sommer, T., Raz, A., and Posner, M. I. (2002). Testing the efficiency and independence of attentional networks. J. Cogn. Neurosci. 14, 340-347. doi: 10.1162/089892902317361886

Ferguson, C. J., and Brent, D. M. (2014). Is the association between children's baby video viewing and poor language development robust? A reanalysis of Zimmerman, Christakis, and Meltzoff (2007). Dev. Psychol. 50, 129-137. doi: $10.1037 / \mathrm{a} 0033628$

Foster, E. M., and Watkins, S. (2010). The value of reanalysis: TV viewing and attention problems. Child Dev. 81, 368-375. doi: 10.1111/j.1467-8624.2009. 01400.x

Hair, J. F. Jr., Anderson, R. E., Tatham, R. L., and Black, W. C. (1995). Multivariate Data Analysis, 3rd Edn. New York, NY: Macmillan.

Hayes, A. F. (2009). Beyond Baron and Kenny: statistical mediation analysis in the new millennium. Commun. Monogr. 76, 408-420. doi: 10.1080/ 03637750903310360

Hayes, A. F. (2015). An index and test of linear moderated mediation. Multivariate Behav. Res. 50, 1-22. doi: 10.1080/00273171.2014.962683

He, C. (2015). Report on China Labor-Force Dynamic Survey. Beijing: Social Sciences Literature Press.

Hofferth, S. L. (2010). Home media and children's achievement and behavior. Child Dev. 81, 1598-1619. doi: 10.1111/j.1467-8624.2010.01494.x

Jensen, P. S., Mrazek, D., Knapp, P. K., Steinberg, L., Pfeffer, C., Schowalter, J., et al. (1997). Evolution and revolution in child psychiatry: ADHD as a disorder of adaptation. J. Am. Acad. Child Adolesc. Psychiatry 36, 1672-1681. doi: 10.1097/ 00004583-199712000-00015

Kirkorian, H. L., and Anderson, D. R. (2009). "Learning from educational media," in The Handbook of Children, Media, and Development, eds S. L. Calvert and B. J. Wilson (Malden, MA: John Wiley \& Sons), 188-213.

Lan, X., Ponitz, C. C., Miller, K. F., Li, S., Kai, C., Perry, M., et al. (2009). Keeping their attention: classroom practices associated with behavioral engagement in first grade mathematics classes in China and the United States. Early Child. Res. Q. 24, 198-211. doi: 10.1016/j.ecresq.2009.03.002

Landhuis, C. E., Poulton, R., Welch, D., and Hancox, R. J. (2007). Does childhood television viewing lead to attention problems in adolescence? Results from a prospective longitudinal study. Pediatrics 120, 532-537. doi: 10.1542/peds. 2007-0978

Li, H., Zhou, Z., and Xiangping, W. (2014). Investigation of the media use among children aged 3 to 6 years. Shanghai Res. Educ. 5, 57-59.

Lillard, A., and Peterson, J. (2011). The immediate impact of different types of television on young children's executive function. Pediatrics 128, 644-649. doi: 10.1542/peds.2010-1919

Lillard, A. S., Drell, M. B., Richey, E. M., Boguszewski, K., and Smith, E. D. (2015a). Further examination of the immediate impact of television on children's executive function. Dev. Psychol. 51, 792-805. doi: 10.1037/a0039097 
Lillard, A. S., Hui, L., and Boguszewski, K. (2015b). Television and children's executive function. Adv. Child Dev. Behav. 48, 219-248. doi: 10.1016/bs.acdb. 2014.11.006

Linebarger, D. L., Barr, R., Lapierre, M. A., and Piotrowski, J. T. (2014). Associations between parenting, media use, cumulative risk, and children's executive functioning. J. Dev. Behav. Pediatr. 35, 367-377. doi: 10.1097/DBP. 0000000000000069

Mackinnon, D. P., Fairchild, A. J., and Fritz, M. S. (2007). Mediation analysis. Annu. Rev. Psychol. 58, 593-614. doi: 10.1146/annurev.psych.58.110405.085542

McHale, S. M., Dotterer, A., and Kim, J.-Y. (2009). An ecological perspective on the media and youth development. Am. Behav. Sci. 52, 1186-1203. doi: 10.1177/ 0002764209331541

Miller, E. K., and Cohen, J. D. (2001). An integrative theory of prefrontal cortex function. Annu. Rev. Neurosci. 24, 167-202. doi: 10.1146/annurev.neuro.24. 1.167

Miller, M. R., Giesbrecht, G. F., Müller, U., McInerney, R. J., and Kerns, K. A. (2012). A latent variable approach to determining the structure of executive function in preschool children. J. Cogn. Dev. 13, 395-423. doi: 10.1080/ 15248372.2011.585478

Nathanson, A. I., Aladé, F., Sharp, M. L., Rasmussen, E. E., and Christy, K. (2014). The relation between television exposure and executive function among preschoolers. Dev. Psychol. 50, 1497-1506. doi: 10.1037/a0035714

Nikkelen, S. W., Valkenburg, P. M., Huizinga, M., and Bushman, B. J. (2014). Media use and ADHD-related behaviors in children and adolescents: a meta-analysis. Dev. Psychol. 50, 2228-2241. doi: 10.1037/a0037318

Obel, C., Henriksen, T. B., Dalsgaard, S., Linnet, K. M., Skajaa, E., Thomsen, P. H., et al. (2004). Does children's watching of television cause attention problems? Retesting the hypothesis in a Danish cohort. Pediatrics 114, 1372-1373. doi: 10.1542/peds.2004-0954

Posner, M. I., and Rothbart, M. K. (2007). Research on attention networks as a model for the integration of psychological science. Annu. Rev. Psychol. 58, 1-23. doi: 10.1146/annurev.psych.58.110405.085516

Preacher, K. J., Rucker, D. D., and Hayes, A. F. (2007). Addressing moderated mediation hypotheses: theory, methods, and prescriptions. Multivariate Behav. Res. 42, 185-227. doi: 10.1080/00273170701341316

Razel, M. (2001). The complex model of television viewing and educational achievement. J. Educ. Res. 94, 371-379. doi: 10.1080/00220670109598774

Sabbagh, M. A., Xu, F., Carlson, S. M., Moses, L. J., and Lee, K. (2006). The development of executive functioning and theory of mind. A comparison of Chinese and U.S. preschoolers. Psychol. Sci. 17, 74-81. doi: 10.1111/j.1467-9280. 2005.01667.x

Salomon, G. (1977). Effects of encouraging Israeli mothers to co-observe" Sesame Street" with their five-year-olds. Child Dev. 48, 1146-1151. doi: 10.2307/ 1128378

Sang, B., and Miao, X. (1990). Peabody picture vocabulary test - revised (PPVT-R): the revised norm of Shanghai. Psychol. Sci. 5, 22-27.

Sarsour, K., Sheridan, M., Jutte, D., Nurujeter, A., Hinshaw, S., and Boyce, W. T. (2011). Family socioeconomic status and child executive functions: the roles of language, home environment, and single parenthood. J. Int. Neuropsychol. Soc. 17, 120-132. doi: 10.1017/S1355617710001335
Stevens, T., and Mulsow, M. (2006). There is no meaningful relationship between television exposure and symptoms of attention-deficit/hyperactivity disorder. Pediatrics 117, 665-672. doi: 10.1542/peds.2005-0863

Swing, E. L., Gentile, D. A., Anderson, C. A., and Walsh, D. A. (2010). Television and video game exposure and the development of attention problems. Pediatrics 126, 214-221. doi: 10.1542/peds.2009-1508

Talwar, V., Carlson, S. M., and Lee, K. (2011). Effects of a punitive environment on children's executive functioning: a natural experiment. Soc. Dev. 20, 805-824. doi: 10.1111/j.1467-9507.2011.00617.x

Valkenburg, P. M., Krcmar, M., Peeters, A. L., and Marseille, N. M. (1999). Developing a scale to assess three styles of television mediation: "Instructive mediation," "restrictive mediation," and "social coviewing". J. Broadcast. Electron. Med. 43, 52-66. doi: 10.1080/08838159909364474

Wang, J., and Mao, S. (1996). Culture and the kindergarten curriculum in the People's Republic of China. Early Child Dev. Care 123, 143-156. doi: 10.1080/ 0300443961230110

Wartella, E., Richert, R. A., and Robb, M. B. (2010). Babies, television and videos: How did we get here? Dev. Rev. 30, 116-127. doi: 10.1016/j.dr.2010.03.008

Wiebe, S. A., Espy, K. A., and Charak, D. (2008). Using confirmatory factor analysis to understand executive control in preschool children: I. Latent structure. Dev. Psychol. 44, 575-587. doi: 10.1037/0012-1649.44.2.575

Williams, P. A. (1982). The impact of leisure-time television on school learning: a research synthesis. Am. Educ. Res. J. 19, 19-50. doi: 10.3102/ 00028312019001019

Willoughby, M. T., Wirth, R. J., and Blair, C. B. (2011). Contributions of modern measurement theory to measuring executive function in early childhood: an empirical demonstration. J. Exp. Child Psychol. 108, 414-435. doi: 10.1016/j. jecp.2010.04.007

Wright, J. C., Huston, A. C., Murphy, K. C., St. Peters, M., Pinin, M., Scantlin, R. et al. (2001). The relations of early television viewing to school readiness and vocabulary of children from low-income families: the Early Window project. Child Dev. 72, 1347-1366. doi: 10.1111/1467-8624.t01-1-00352

Wu, C. S. T., Fowler, C., Lam, W. Y. Y., Wong, H. T., Wong, C. H. M., and Loke, A. Y. (2014). Parenting approaches and digital technology use of preschool age children in a Chinese community. Italian J. Pediatr. 40:44. doi: 10.1186/18247288-40-44

Zimmerman, F. J., and Christakis, D. A. (2007). Associations between content types of early media exposure and subsequent attentional problems. Pediatrics 120, 986-992. doi: 10.1542/peds.2006-3322

Conflict of Interest Statement: The authors declare that the research was conducted in the absence of any commercial or financial relationships that could be construed as a potential conflict of interest.

Copyright (c) 2017 Yang, Chen, Wang and Zhu. This is an open-access article distributed under the terms of the Creative Commons Attribution License (CC BY). The use, distribution or reproduction in other forums is permitted, provided the original author(s) or licensor are credited and that the original publication in this journal is cited, in accordance with accepted academic practice. No use, distribution or reproduction is permitted which does not comply with these terms. 